\title{
An explicit formula for Bell numbers in terms of Stirling numbers and hypergeometric functions
}

\author{
Bai-Ni Guo ${ }^{1, *} \&$ Feng $\mathbf{Q i}^{2,3}$ \\ ${ }^{1}$ School of Mathematics and Informatics, Henan Polytechnic University, Jiaozuo City, Henan Province, 454010, China \\ ${ }^{2}$ College of Mathematics, Inner Mongolia University for Nationalities, \\ Tongliao City, Inner Mongolia Autonomous Region, 028043, China \\ ${ }^{3}$ Department of Mathematics, College of Science, Tianjin Polytechnic University, Tianjin City, 300387, China \\ *Corresponding author'se-mail: bai.ni.guo@gmail.com,bai.ni.guo@hotmail.com \\ * Corresponding author's URL: http: //www. researchgate. net/profile/Bai-Ni_Guo/
}

Copyright (C)2014 Bai-Ni Guo and Feng Qi. This is an open access article distributed under the Creative Commons Attribution License, which permits unrestricted use, distribution, and reproduction in any medium, provided the original work is properly cited.

\begin{abstract}
In the paper, by two methods, the authors find an explicit formula for computing Bell numbers in terms of Kummer confluent hypergeometric functions and Stirling numbers of the second kind. Moreover, the authors supply an alternative proof of the well-known "triangular" recurrence relation for Stirling numbers of the second kind. In a remark, the authors reveal the combinatorial interpretation of the special values for Kummer confluent hypergeometric functions and the total sum of Lah numbers.
\end{abstract}

Keywords: explicit formula; Bell number; confluent hypergeometric function of the first kind; Stirling number of the second kind; combinatorial interpretation; alternative proof; recurrence relation; polylogarithm

MSC: Primary 11B73; Secondary 33B10, 33C15

\section{Introduction}

In combinatorics, Bell numbers, usually denoted by $\mathrm{B}_{n}$ for $n \in\{0\} \cup \mathbb{N}$, count the number of ways a set with $n$ elements can be partitioned into disjoint and non-empty subsets. These numbers have been studied by mathematicians since the 19th century, and their roots go back to medieval Japan, but they are named after Eric Temple Bell, who wrote about them in the 1930s. Every Bell number $\mathrm{B}_{n}$ may be generated by

$e^{e^{x}-1}=\sum_{k=0}^{\infty} \mathrm{B}_{k} \frac{x^{k}}{k !}$

or, equivalently, by

$e^{e^{-x}-1}=\sum_{k=0}^{\infty}(-1)^{k} \mathrm{~B}_{k} \frac{x^{k}}{k !}$. 
In combinatorics, Stirling numbers arise in a variety of combinatorics problems. They are introduced in the eighteen century by James Stirling. There are two kinds of Stirling numbers: Stirling numbers of the first kind and Stirling numbers of the second kind. Every Stirling number of the second kind, usually denoted by $S(n, k)$, is the number of ways of partitioning a set of $n$ elements into $k$ nonempty subsets, may be computed by

$S(n, k)=\frac{1}{k !} \sum_{i=0}^{k}(-1)^{i}\left(\begin{array}{c}k \\ i\end{array}\right)(k-i)^{n}$,

and may be generated by

$\frac{\left(e^{x}-1\right)^{k}}{k !}=\sum_{n=k}^{\infty} S(n, k) \frac{x^{n}}{n !}, \quad k \in\{0\} \cup \mathbb{N}$.

In the theory of special functions, the hypergeometric functions are denoted and defined by

${ }_{p} F_{q}\left(a_{1}, \ldots, a_{p} ; b_{1}, \ldots, b_{q} ; x\right)=\sum_{n=0}^{\infty} \frac{\left(a_{1}\right)_{n} \cdots\left(a_{p}\right)_{n}}{\left(b_{1}\right)_{n} \cdots\left(b_{q}\right)_{n}} \frac{x^{n}}{n !}$

for $b_{i} \notin\{0,-1,-2, \ldots\}$ and $p, q \in \mathbb{N}$, where $(a)_{0}=1$ and $(a)_{n}=a(a+1) \cdots(a+n-1)$ for $n \in \mathbb{N}$ and any complex number $a$ is called the rising factorial. Specially, the series

${ }_{1} F_{1}(a ; b ; z)=\sum_{k=0}^{\infty} \frac{(a)_{k}}{(b)_{k}} \frac{z^{k}}{k !}$

is called Kummer confluent hypergeometric function.

In combinatorics or number theory, it is common knowledge that Bell numbers $\mathrm{B}_{n}$ may be computed in terms of Stirling numbers of the second kind $S(n, k)$ by

$\mathrm{B}_{n}=\sum_{k=1}^{n} S(n, k)$.

In this paper, by two methods, we will find a new explicit formula for computing Bell numbers $\mathrm{B}_{n}$ in terms of Kummer confluent hypergeometric functions ${ }_{1} F_{1}(k+1 ; 2 ; 1)$ and Stirling numbers of the second kind $S(n, k)$ as follows.

Theorem 1. For $n \in \mathbb{N}$, Bell numbers $\mathrm{B}_{n}$ may be expressed as

$\mathrm{B}_{n}=\frac{1}{e} \sum_{k=1}^{n}(-1)^{n-k} k !_{1} F_{1}(k+1 ; 2 ; 1) S(n, k)$.

It is well known in combinatorics that Stirling numbers of the second kind $S(n, k)$ satisfy the "triangular" recurrence relation

$S(n, k)=S(n-1, k-1)+k S(n-1, k), \quad k, n \geq 1$

and

$S(n, 0)=S(0, k)=0, \quad S(0,0)=1$.

See [2, p. 208, Theorem A]. In [2, pp. 208-209], the author provided an analytical proof and a combinatorial proof of (1.9). In Section 3 of this paper, we will supply an alternative proof of (1.9) by virtue of some properties of the polylogarithm $\operatorname{Li}_{n}(z)$ which may be defined by

$\operatorname{Li}_{n}(z)=\sum_{k=1}^{\infty} \frac{z^{k}}{k^{n}}$

over the open unit disk in the complex plane $\mathbb{C}$ and extended to the whole complex plane $\mathbb{C}$ uniquely via analytic continuation. 


\section{Proofs of Theorem 1}

We now start out to verify Theorem 1 as follows.

First proof. Among other things, it was obtained in [8, Theorem 1.2] that the function

$H_{k}(z)=e^{1 / z}-\sum_{m=0}^{k} \frac{1}{m !} \frac{1}{z^{m}}$

for $k \in\{0\} \cup \mathbb{N}$ and $z \neq 0$ has the integral representation

$H_{k}(z)=\frac{1}{k !(k+1) !} \int_{0}^{\infty}{ }_{1} F_{2}(1 ; k+1, k+2 ; t) t^{k} e^{-z t} \mathrm{~d} t, \quad \Re(z)>0$.

See also [6, Section 1.2] and [7, Lemma 2.1]. When $k=0$, the integral representation (2.2) becomes

$e^{1 / z}=1+\int_{0}^{\infty} \frac{I_{1}(2 \sqrt{t})}{\sqrt{t}} e^{-z t} \mathrm{~d} t, \quad \Re(z)>0$,

where $I_{\nu}(z)$ stands for the modified Bessel function of the first kind

$I_{\nu}(z)=\sum_{k=0}^{\infty} \frac{1}{k ! \Gamma(\nu+k+1)}\left(\frac{z}{2}\right)^{2 k+\nu}$

for $\nu \in \mathbb{R}$ and $z \in \mathbb{C}$, see $[1$, p. 375, 9.6.10], and $\Gamma$ represents the classical Euler gamma function which may be defined by

$\Gamma(z)=\int_{0}^{\infty} t^{z-1} e^{-t} \mathrm{~d} t, \quad \Re z>0$,

see $\left[1\right.$, p. 255]. Replacing $z$ by $e^{x}$ in (2.3) gives

$e^{1 / e^{x}}=e^{e^{-x}}=1+\int_{0}^{\infty} \frac{I_{1}(2 \sqrt{t})}{\sqrt{t}} e^{-e^{x} t} \mathrm{~d} t$.

Differentiating $n \geq 1$ times with respect to $x$ on both sides of (2.6) and (1.2) gives

$\frac{\mathrm{d}^{n} e^{e^{-x}}}{\mathrm{~d} x^{n}}=\int_{0}^{\infty} \frac{I_{1}(2 \sqrt{t})}{\sqrt{t}} \frac{\mathrm{d}^{n} e^{-e^{x} t}}{\mathrm{~d} x^{n}} \mathrm{~d} t$

and

$\frac{\mathrm{d}^{n} e^{e^{-x}}}{\mathrm{~d} x^{n}}=e \sum_{k=n}^{\infty}(-1)^{k} \mathrm{~B}_{k} \frac{x^{k-n}}{(k-n) !}$.

From (2.7) and (2.8), it follows that

$e \sum_{k=n}^{\infty}(-1)^{k} \mathrm{~B}_{k} \frac{x^{k-n}}{(k-n) !}=\int_{0}^{\infty} \frac{I_{1}(2 \sqrt{t})}{\sqrt{t}} \frac{\mathrm{d}^{n} e^{-e^{x} t}}{\mathrm{~d} x^{n}} \mathrm{~d} t$.

Taking $x \rightarrow 0$ in the above equation yields

$(-1)^{n} e \mathrm{~B}_{n}=\int_{0}^{\infty} \frac{I_{1}(2 \sqrt{t})}{\sqrt{t}} \lim _{x \rightarrow 0} \frac{\mathrm{d}^{n} e^{-e^{x} t}}{\mathrm{~d} x^{n}} \mathrm{~d} t$.

In combinatorics, it is well known that Bell polynomials of the second kind, or say, the partial Bell polynomials, denoted by $\mathrm{B}_{n, k}\left(x_{1}, x_{2}, \ldots, x_{n-k+1}\right)$, may be defined by

$$
\mathrm{B}_{n, k}\left(x_{1}, x_{2}, \ldots, x_{n-k+1}\right)=\sum_{\substack{1 \leq i \leq n, \ell_{i} \in\{0\} \cup \mathbb{N} \\ \sum_{i=1}^{n} i \ell_{i}=n \\ \sum_{i=1}^{n} \ell_{i}=k}} \frac{n !}{\prod_{i=1}^{n-k+1} \ell_{i} !} \prod_{i=1}^{n-k+1}\left(\frac{x_{i}}{i !}\right)^{\ell_{i}}
$$


for $n \geq k \geq 0$, see $[2$, p. 134 , Theorem A], and satisfy

$\mathrm{B}_{n, k}\left(a b x_{1}, a b^{2} x_{2}, \ldots, a b^{n-k+1} x_{n-k+1}\right)=a^{k} b^{n} \mathrm{~B}_{n, k}\left(x_{1}, x_{n}, \ldots, x_{n-k+1}\right)$

and

$\mathrm{B}_{n, k}(1,1, \ldots, 1)=S(n, k)$

see [2, p. 135], where $a$ and $b$ are any complex numbers. The well-known Faà di Bruno formula may be described in terms of Bell polynomials of the second kind $\mathrm{B}_{n, k}\left(x_{1}, x_{2}, \ldots, x_{n-k+1}\right)$ by

$\frac{\mathrm{d}^{n}}{\mathrm{~d} x^{n}} f \circ g(x)=\sum_{k=1}^{n} f^{(k)}(g(x)) \mathrm{B}_{n, k}\left(g^{\prime}(x), g^{\prime \prime}(x), \ldots, g^{(n-k+1)}(x)\right)$,

see [2, p. 139, Theorem C]. By Faà di Bruno formula (2.13) and the identities (2.11) and (2.12), we have

$$
\begin{aligned}
& \frac{\mathrm{d}^{n} e^{-e^{x} t}}{\mathrm{~d} x^{n}}=\sum_{k=1}^{n} e^{-e^{x} t} \mathrm{~B}_{n, k}\left(-e^{x} t,-e^{x} t, \ldots,-e^{x} t\right)=e^{-e^{x} t} \sum_{k=1}^{n}\left(-e^{x} t\right)^{k} \mathrm{~B}_{n, k}(1,1, \ldots, 1) \\
& =e^{-e^{x} t} \sum_{k=1}^{n}\left(-e^{x} t\right)^{k} S(n, k) \rightarrow e^{-t} \sum_{k=1}^{n}(-t)^{k} S(n, k)
\end{aligned}
$$

as $x \rightarrow 0$. Substituting the above limit into (2.9) leads to

$\mathrm{B}_{n}=\frac{1}{e} \sum_{k=1}^{n}(-1)^{n-k} S(n, k) \int_{0}^{\infty} I_{1}(2 \sqrt{t}) t^{k-1 / 2} e^{-t} \mathrm{~d} t=\frac{1}{e} \sum_{k=1}^{n}(-1)^{n-k} S(n, k) k !_{1} F_{1}(k+1 ; 2 ; 1)$.

The required proof is complete.

Second proof. From the definition of the exponential function and the formula

$\frac{k !}{z^{k+1}}=\int_{0}^{\infty} e^{-z t} t^{k} \mathrm{~d} t$

it was obtained that

$e^{1 / z}=1+\int_{0}^{\infty} e^{-z t} \sum_{k=0}^{\infty} \frac{t^{k}}{k !(k+1) !} \mathrm{d} t$.

Substituting $z$ with $e^{x}$ and using the exponential generating function for Bell polynomials

$\sum_{n=0}^{\infty} \frac{\mathrm{B}_{n}(t)}{n !} x^{n}=e^{t\left(e^{x}-1\right)}$

lead to

$e \sum_{n=0}^{\infty} \frac{\mathrm{B}_{n}(1)}{n !}(-x)^{n}=1+\int_{0}^{\infty} e^{-t} \sum_{n=0}^{\infty} \frac{\mathrm{B}_{n}(-t)}{n !} x^{n} \sum_{k=0}^{\infty} \frac{t^{k}}{k !(k+1) !} \mathrm{d} t$.

Equating coefficients of $x^{n}$ and using the fact that

$\mathrm{B}_{n}(t)=\sum_{k=0}^{\infty} S(n, k) t^{k}$

and $\mathrm{B}_{n}(1)=\mathrm{B}_{n}$, the equation (1.8) follows. 


\section{An alternative proof of the recurrence relation (1.9)}

In [11], the formula

$\sum_{k=1}^{n} S(n, k)(k-1) ! z^{k}=(-1)^{n} \operatorname{Li}_{1-n}\left(1+\frac{1}{z}\right)$

for $n \geq 2$ is listed. Making use of

$\frac{\mathrm{d}}{\mathrm{d} x} \operatorname{Li}_{n}(x)=\frac{1}{x} \operatorname{Li}_{n-1}(x)$,

see [10], and differentiating with respect to $z=x$ on both sides of (3.1) give

$\sum_{k=1}^{n} S(n, k) k ! x^{k-1}=(-1)^{n+1} \frac{1}{(1+x) x} \operatorname{Li}_{-n}\left(1+\frac{1}{x}\right)$

which may be reformulated as

$(1+x) \sum_{k=1}^{n} S(n, k) k ! x^{k}=(-1)^{n+1} \mathrm{Li}_{-n}\left(1+\frac{1}{x}\right)$.

Comparing (3.1) with (3.3) yields

$\sum_{k=1}^{n+1} S(n+1, k)(k-1) ! x^{k}=(1+x) \sum_{k=1}^{n} S(n, k) k ! x^{k}=\sum_{k=1}^{n} S(n, k) k ! x^{k}+\sum_{k=2}^{n+1} S(n, k-1)(k-1) ! x^{k}$

$=S(n, 1) x+S(n, n) n ! x^{n+1}+\sum_{k=2}^{n}[k S(n, k)+S(n, k-1)](k-1) ! x^{k}$

$=x+n ! x^{n+1}+\sum_{k=2}^{n}[k S(n, k)+S(n, k-1)](k-1) ! x^{k}$.

This implies that $S(n, 1)=S(n, n)=1$ and

$S(n+1, k)=S(n, k-1)+k S(n, k), \quad k, n \geq 2$.

Thus, we recover the recurrence relation (1.9).

\section{Remarks}

Remark 1. In view of the formula (1.7), it is not clear why the new formula (1.8) is of interest for computing $B_{n}$. Changing the point of view, it could be of interest to know that the sum on the right side of (1.8) evaluates to Bell number $B_{n}$.

Remark 2. The equation (1.8) may be rewritten as

$\sum_{k=1}^{n}(-1)^{n-k} a_{k} S(n, k)=\mathrm{B}_{n}$

where $a_{k}$ is sequence A000262 in the Online Encyclopedia of Integer Sequences. Such a sequence $a_{k}$ has a nice combinatorial interpretation: it counts "the sets of lists, or the number of partitions of $\{1,2 \ldots, k\}$ into any number of lists, where a list means an ordered subset." In [4], it was obtained that

$a_{k}=\sum_{\ell=1}^{k} L(k, \ell), \quad k \in \mathbb{N}$.

See also [3]. This reveals the combinatorial interpretation of the special sequence $k !_{1} F_{1}(k+1 ; 2 ; 1)$ and the total sum $\mathcal{L}_{k}=\sum_{\ell=1}^{k} L(k, \ell)$ of Lah numbers $L(k, \ell)$.

Remark 3. By the way, the authors of the paper [9] discovered some new results on the polylogarithm $\operatorname{Li}_{n}(z)$.

Remark 4. This paper is a revised and extended version of the preprint [5]. 


\section{References}

[1] M. Abramowitz and I. A. Stegun (Eds), Handbook of Mathematical Functions with Formulas, Graphs, and Mathematical Tables, National Bureau of Standards, Applied Mathematics Series 55, 10th printing, Dover Publications, 1972.

[2] L. Comtet, Advanced Combinatorics: The Art of Finite and Infinite Expansions, Revised and Enlarged Edition, D. Reidel Publishing Co., 1974.

[3] B.-N. Guo and F. Qi, Some integral representations and properties of Lah numbers, available online at http://arxiv.org/abs/1402.2367.

[4] F. Qi, An explicit formula for computing Bell numbers in terms of Lah and Stirling numbers, available online at http://arxiv.org/abs/1401.1625.

[5] F. Qi, An explicit formula for Bell numbers in terms of Stirling numbers and hypergeometric functions, available online at http://arxiv.org/abs/1402.2361.

[6] F. Qi, Properties of modified Bessel functions and completely monotonic degrees of differences between exponential and trigamma functions, Math. Inequal. Appl. 18 (2015), in press; Available online at http://arxiv.org/abs/1302.6731.

[7] F. Qi and C. Berg, Complete monotonicity of a difference between the exponential and trigamma functions and properties related to a modified Bessel function, Mediterr. J. Math. 10 (2013), no. 4, 1685-1696; Available online at http://dx.doi.org/10.1007/s00009-013-0272-2.

[8] F. Qi and S.-H. Wang, Complete monotonicity, completely monotonic degree, integral representations, and an inequality related to the exponential, trigamma, and modified Bessel functions, Glob. J. Math. Anal. 2 (2014), no. 3, 91-97; Available online at http://dx.doi.org/10.14419/gjma.v2i3.2919.

[9] C.-F. Wei and B.-N. Guo, Complete monotonicity of functions connected with the exponential function and derivatives, Abstr. Appl. Anal. 2014 (2014), Article ID 851213, 5 pages; Available online at http://dx.doi.org/10.1155/2014/851213.

[10] E. W. Weisstein, Polylogarithm, MathWorld-A Wolfram Web Resource; Available online at http://mathworld.wolfram.com/Polylogarithm.html.

[11] E. W. Weisstein, Stirling Number of the Second Kind, MathWorld-A Wolfram Web Resource; Available online at http://mathworld.wolfram.com/StirlingNumberoftheSecondKind.html. 